\title{
Topology association analysis in weighted protein interaction network for gene prioritization
}

\author{
Shunyao $\mathrm{Wu}^{\mathrm{a}}$, Fengjing Shao ${ }^{\mathrm{a}, *}$, Qi Zhang ${ }^{\mathrm{a}}$, Jun $\mathrm{Ji}^{\mathrm{a}}$, Shaojie Xu${ }^{\mathrm{a}}$, Rencheng \\ Sun $^{\mathrm{a}}$, Gengxin Sun ${ }^{\mathrm{a}}$, Xiangjun $\mathrm{Du}^{\mathrm{a}}$, Yi Sui ${ }^{\mathrm{a}}$ \\ ${ }^{a}$ Qingdao University, Qingdao 266071, China
}

\begin{abstract}
Although lots of algorithms for disease gene prediction have been proposed, the weights of edges are rarely taken into account. In this paper, the strengths of topology associations between disease and essential genes are analyzed in weighted protein interaction network. Empirical analysis demonstrates that compared to other genes, disease genes are weakly connected with essential genes in protein interaction network. Based on this finding, a novel global distance measurement for gene prioritization with weighted protein interaction network is proposed in this paper. Positive and negative flow is allocated to disease and essential genes respectively. Additionally network propagation model is extended for weighted network. Experimental results on 110 diseases verify the effectiveness and potential of the proposed measurement. Moreover, weak links play more important role than strong links for gene prioritization, which is meaningful to deeply understand protein interaction network.
\end{abstract}

Keywords:

weighted protein interaction network, disease genes, essential genes, topology association, gene prioritization

\section{Introduction}

The flourish of disease gene prediction is generally recognized as the breakthrough that discovers pathogenesis and promotes the diagnosis quality.

\footnotetext{
${ }^{*}$ Corresponding author

Email address: sfj@qdu.edu.cn. (Fengjing Shao)
} 
However, it still depletes inevitably time and resources with biomedical experiments and clinical evidence. Due to the fulfill of human genome project, the explosions of omics data, such as metabolic data, proteomic data and protein interactions, offer the brand-new opportunity to design intelligent models and reveal the disease mechanism [1, 2].

In the beginning, gene classification was proposed to predict disease genes based on machine learning theory [3-9]. Generally, gene classification trained models via sequence-based features and topological features, and then automatically identified candidate disease genes. The main defect lied in negative samples selected from unknown genes, which would debase the performance of classifiers [5, 9].

To overcome above challenge, gene prioritization became another potential strategy for disease gene prediction [10-15]. Given one hereditary disease and its known disease genes, the potential disease genes could be searched out according to the topological similarities between unknown genes and known disease genes. Molecule networks, such as protein interaction networks, were utilized to uncover the mechanics of diseases, for molecule interactions usually reflected function linkages among molecules [16]. Perturbations of molecule networks probably led to human diseases [17, 18], and neighbors of disease genes were likely to cause the same or similar diseases [19]. Overall, network-based methods for gene prioritization have been well studied by complex network theory $[11,20,21]$, while some problems are still in suspense. For instance, existing methods are confined to detect potential disease genes within neighborhoods of known disease genes [21], or even mistakes non-disease hub proteins as potential disease genes [15].

Majority studies on gene prioritization are concerned with un-weighted protein interaction networks. Besides that, empirical analyses for the topological properties of disease proteins also mainly focus on them. More precisely, these networks are constructed with strong protein interactions, which are specified by a given protein interaction threshold. Obviously the analysis complexity is considerably minimized at expense of weak protein interactions. Currently, weighted molecule networks have stepped into the public view by degrees $[22,31,32]$. Weak links among the neural network count little for link prediction [22], whereas they are essential to stabilize the conjunctions between functional modules in molecule networks [23]. Therefore, the strengths of molecular interactions remain to be explored for deeply understanding protein interaction networks.

In this paper, topology associations between disease and essential genes 
are analyzed in the weighted protein interaction network. Since empirical analysis exhibits disease proteins are weakly connected to essential proteins, network propagation is extended for weighted protein interaction network. This extended model superiorly outperforms the previous methods supported by experimental results. In addition, this paper states that weak links impact disease gene prediction. Compared to the previous methods, network propagation with dual flow could make better use of weak links.

\section{Materials and Methods}

\subsection{Human disease gene list and housekeeping gene list}

The disease gene list is downloaded from the Online Mendelian Inheritance in Man database (OMIM) [24]. 2931 disease genes verified by the presence of a mutation with tag ' 3 ' are selected from 6285 entries.

Housekeeping genes are obtained from the research of Chang et al. [25] They are universally expressed in normal tissues or cells and vital to maintaining fundamental life activities. Thus, housekeeping genes can be deemed as essential genes [26].

\subsection{Gene-disease associations}

110 hereditary diseases and corresponding disease genes are obtained from Kohler et al.'s work ${ }^{1}$ [11]. They have collected the associations between genetic diseases and disease genes from OMIM, domain knowledge and medicinal literatures. The collection contains 794 gene-disease associations, but only involves 681 unique genes (one gene may cause a few disease).

\subsection{Protein interactions}

The human protein interactions are downloaded from STRING database (version 9.05) [27], which provides a score to evaluate the reliablity between any two interactors. The STRING database collects protein interactions by utilizing various different kinds of active prediction methods including Neighborhood, Gene Fusion, Text Mining, Co-occurrence, Co-expression, Experiments and Databases [27]. This paper extracts direct human binding interactions from STRING action database. All the binding interactions

\footnotetext{
${ }^{1}$ http://www.cell.com/cms/attachment/2024884754/2044549085/mmc1.zip
} 
are physical interactions and gained by Experiments. The protein interaction network is constructed with 14018 interactors and 164,087 interactions inferred from experimental data.

Table 1: Statistics of interactors in the protein interaction network.

\begin{tabular}{|c|c|}
\hline & Number of Interactors \\
\hline$D$ & 2551 \\
\hline$E$ & 1904 \\
\hline$E \cap D$ & 295 \\
\hline$D^{-}$ & 2256 \\
\hline$E^{-}$ & 1609 \\
\hline$O$ & 9858 \\
\hline
\end{tabular}

In this paper, essential genes and disease genes are annotated as $E$ and $D$ respectively, and the remaining genes $(O=\neg(E \bigcup D))$ are treated as other genes. Table 1 lists the statistics of different types of interactors in the protein interaction network constructed via the STRING database. There exists a small intersection between essential genes and disease genes, which probably influences the conclusions of topology associations [8]. Thus, essential and disease genes $(E \bigcap D)$ are eliminated from our analysis. For the sake of brevity, $\neg D \bigcap E$ and $\neg E \bigcap D$ are denoted by $E^{-}$and $D^{-}$respectively.

\subsection{Node similarity in weighted networks}

Node similarity has been well studied in un-weighted networks, which is not the case in weighted networks. As one representative, Dijkstra's shortest path algorithm evaluates topology associations between nodes in weighted network. Inspired by Symeonidis et al. [28], this paper proposes a variant of Dijkstra's algorithm named maximum transitive similarity to calculate node similarities in weighted networks.

Weighted network with $n$ nodes is denoted as $G=(V, L, W)$. Here, node set $V=\left\{v_{1}, v_{2}, \ldots v_{n}\right\}$, edge set $L=\left\{l_{i j}=\left(v_{i}, v_{j}\right) \mid v_{i}, v_{j} \in V\right\}$ and edge weight set $W=\left\{w_{i j} \mid 0<w_{i j} \leq 1, l_{i j} \in L\right\}$. Given two nodes $x$ and $y$ in a weighted network, their similarity is computed as follow

$$
\operatorname{Sim}_{x y}=\left\{\begin{array}{l}
0, \quad \text { if there is no path between } x \text { and } y \\
w_{i j}, \quad \text { if }(x, y) \in L \\
\max _{\mathcal{P}} \prod_{h=1}^{k} w_{v_{h} v_{h+1}}, \quad \text { otherwise }
\end{array}\right.
$$


Here, $v_{1}=x, v_{k+1}=y$, set $\mathcal{P}$ contains all paths from $x$ to $y$, and $v_{h}(h=$ $2, \ldots, k)$ represents nodes passing by one path in $\mathcal{P}$.

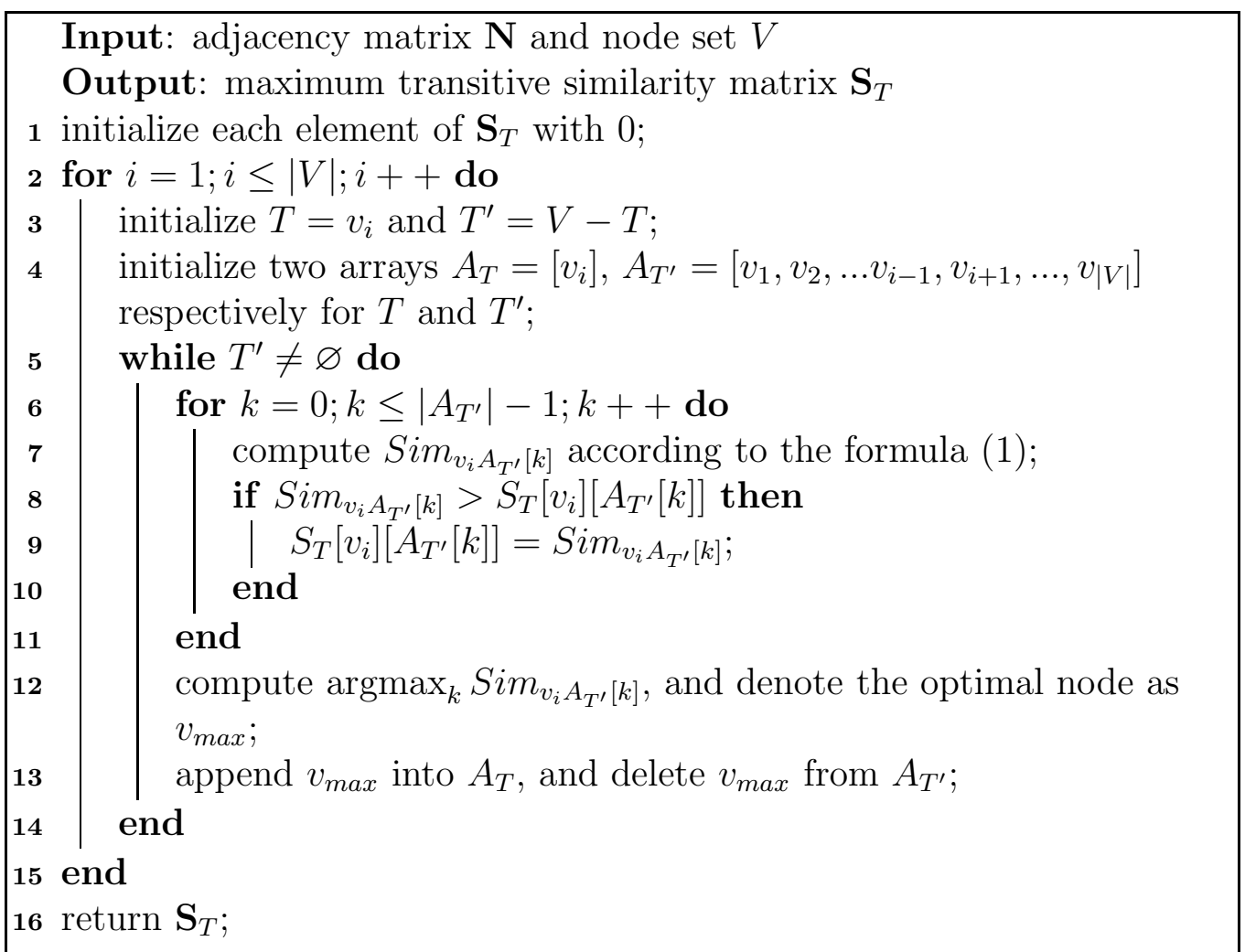

Algorithm 1: A variant of Dijkstra's algorithm for maximum transitive similarity.

To find the optimal solution for $\max _{\mathcal{P}} \prod_{h=1}^{k} w_{v_{h} v_{h+1}}$, Dijkstra's algorithm is modified as illustrated in Algorithm 1. The main change resides in replacing distance with transfer similarity. As clarified in Figure 1, there exist three paths between protein $a$ and $b$, viz. $a b, a c b$ and $a d b$, and the transitive similarities are respectively $0.1,0.35$ and 0.81 . The path with maximum transitive similarity $a c b$ could be gotten by the variant of Dijkstra's Algorithm. 


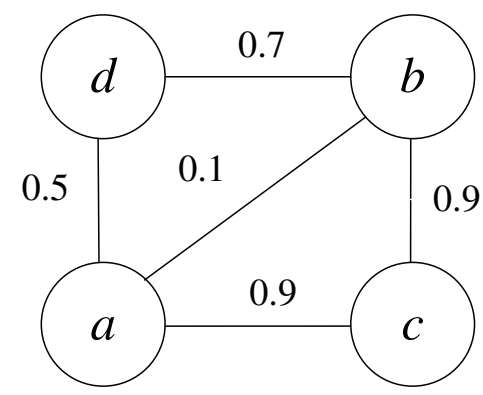

Figure 1: An example for node similarities in a weighted network.

\subsection{Topology index for analyzing the strengths of topology associations}

Wu et al. [15] once defined $Q_{i}^{n}$ ( $n$ neighbors of node $i$ ) with regard to topology associations between disease and essential genes in un-weighted protein interactions, where $Q_{i}^{n}$ stood for a node set including all elements with shortest path length $n$ away from node $i$. Similarly, $Q_{i}^{n}$ is extended for the set in which the hop count from each element to node $i$ is $n$. Given node $i$ and the set $Q_{E^{-}}^{n}\left(Q_{E^{-}}^{n}=Q_{i}^{n} \cap E^{-}\right)$, including non-disease essential proteins in $n$ neighbors of node $i$, the strength of topology associations between them is formulated as follows

$$
s_{E_{i}^{-}}^{n}=\frac{\sum_{j \in Q_{E^{-}}^{n}} \operatorname{Sim}_{i j}}{\sum_{j \in Q_{i}^{n}} \operatorname{Sim}_{i j}}
$$

In this paper, $\left\{s_{E_{i}^{-}}^{n} \mid i \in X\right\}$ is marked as $S_{E^{-} X}^{n}$ with its median as $M d\left(S_{E^{-} X}^{n}\right)$.

\subsection{Gene prioritization with weighted protein interaction network}

As we all known, the typical hypothesis in network medicine is that neighbors of disease genes are likely to cause similar diseases (hypothesis 1). Recently, $\mathrm{Wu}$ et al. [15] posed a new one that if too many non-disease essential proteins existed as neighbors of a candidate protein, the protein was not likely to cause diseases (hypothesis 2). Thus a novel global distance measurement was taken to integrate the two hypotheses for gene prioritization.

In this paper, we consider a hypothesis that if the interaction between a candidate protein and its neighborhood non-disease essential proteins is strong enough, the protein is not likely to cause diseases (hypothesis 3). The network propagation method is employed to exploit the new hypothesis. It can be regarded as a procedural simulation on a weighted network, in which 
each node will pump flow to its direct neighbors in proportion to edge weights during each timestamp. The network propagation model is defined as follows

$$
F^{t+1}=(1-\alpha) \mathbf{N} F^{t}+\alpha Y
$$

Here, $Y$ is priori information for initializing nodes' flow; $F^{t}$ is a vector in which the $i$-th elements holds the flow allocated to node $i$ at timestamp $t$, and $F^{1}=Y$; parameter $\alpha$ controls the prevalence of $Y ; \mathbf{N}$ is the columnnormalized adjacency matrix of the network. Given one unit flow to node $v_{i}$, the flow pump from node $v_{i}$ to its direct neighbor node $v_{j}$ is noted by $N\left(v_{i}, v_{j}\right)=w_{i j} /\left(\sum_{k \in Q_{i}^{1}} w_{i k}\right)$.

Given $F^{t+1}=F^{t}$, the steady-state solution $F^{\infty}$ of equation (3) is

$$
F^{\infty}=\alpha(\mathbf{I}-(1-\alpha) \mathbf{N})^{-1} Y
$$

In this paper, $\alpha(\mathbf{I}-(1-\alpha) \mathbf{N})^{-1}$ is indicated by $\mathbf{M}$ for simplicity. Through setting different initial values of $Y$, various kinds of particular solutions of equation (4) could be obtained.

Assigning +1 unit flow to each disease gene of a certain hereditary disease $h$, the solution named $N P_{D}$ is the typical global distance measurement for disease gene prediction [11]. It reflects the hypothesis 1 . The similarity of candidate gene $x$ with known disease genes is formulated as follow

$$
F^{\infty}=\sum_{y \in T_{h}} M(x, y)
$$

Here, set $T_{h}$ contains all known disease genes of $h$.

To merge the hypothesis 1 and the hypothesis 3, we allot $+\frac{1}{\left|T_{h}\right|}$ flow to each disease gene, while $-\frac{1}{\left|E^{-}\right|}$flow to each non-disease essential gene. Then the solution $N P_{D \& E}$ is composed as

$$
F^{\infty}=\frac{1}{\left|T_{h}\right|} \sum_{y \in T_{h}} M(x, y)-\frac{1}{\left|E^{-}\right|} \sum_{y \in E^{-}} M(x, y)
$$

\subsection{Experimental setup}

We intend to compare our approach $N P_{D \& E}$ with a global distance measurement Network Propagation $\left(N P_{D}\right)[37]$ and three local distance measurements: Direct Neighbors (DN) [19], Shortest Path (SP) [30] and Reliableroute weighted $R A$ index $(r W R A)$ [32]. All the four competing methods 
are designed based on the hypothesis 1 . Specifically, $N P_{D}$ described before is equivalent to Random Walk with Restart [11]; $D N$ searches for candidate disease genes according to direct neighbor counting [16]; $S P$ is another typical method to evaluate the similarity between a candidate gene and any known disease gene; $r W R A$ is a variant of Common Neighbor, and it performs quite well for link prediction in weighted network [32].

Leave-One-Out Cross-Validation is carried out in the experiments. For disease $h$, we select each disease gene of $h$ as a test set in turn, and leave the remaining $\left|T_{h}\right|-1$ disease genes as the training set respectively. Hence we operate trials $\left|T_{h}\right|$ times for $h$. The disease genes outside the protein interaction network are eliminated from the experimental results. To simulate the case of prioritizing genes inside a linkage interval, we follow the literatures $[11,37]$ and select 100 genes closest to the test gene on the same chromosome to construct a candidate gene list (including the test gene). If the test gene has the same flow with some candidate genes, it will rank last of the list. One special case is Leave-One-Out analysis for monogenic diseases with only one disease-causing gene. Only disease gene is the test gene while the training set does not exist, therefore the four competing methods will be invalid. $N P_{D \& E}$ method, by contrast, still works by utilizing non-disease essential genes, although the performance is not ideal.

Enrichment Score and AUC (Area Under Receiver-Operating Characteristics Curve) are employed to evaluate the performance of the measurements for disease genes prediction. Enrichment Score is a typical evaluation index for gene prioritization [11]. For the trial with a disease gene as test gene, if the final flow allocates to the test gene ranked $r_{t h}$, Enrichment Score is $\frac{50}{r}$. Gene prioritization can be deemed as binary classification by setting a rank threshold to divide genes into two categories, disease and non-disease genes $[10,11]$, and $A U C$ is used to estimate the performance of binary classifiers. A unified threshold can be set for all the trials. Candidate genes above the threshold are considered as positive samples (disease genes), while genes below the threshold are negative samples. For a certain threshold, sensitivity and specificity can be evaluated for gene prioritization methods. Sensitivity and specificity are scaled correspondingly by the proportion of the true disease genes above the threshold among the total prioritizations, and the proportion of genes below the threshold among all of the candidate genes. The Receiver-Operating Characteristics curve can be drawn by the Sensitivity versus (1-Specificity) subject to the threshold separating the prediction class. The range of threshold is from 1 to 100, since the length of candidate 
gene list is 100; the total number of prioritizations is 688 , since there exist 688 gene-disease associations for 110 diseases excluding the disease genes outside the protein interaction network.

\section{Results}

\subsection{Analysis of topology associations between diseases and essential genes}

The strengths of topology associations between disease and non-disease essential genes among $n(n \in\{1,2, \ldots, 10\})$ neighbors are compared with those between other and non-disease essential genes, since the maximum hop in the weighted protein interaction is 10. Table 2 lists $\operatorname{Md}\left(S_{E^{-} O}^{n}\right)$ and $M d\left(S_{E^{-} D^{-}}^{n}\right)(n \in 1,2, \ldots, 5)$ in the weighted protein interaction network. When $n \in\{6,7, \ldots, 10\}$, the median values of $M d\left(S_{E^{-} O}^{n}\right)$ and $M d\left(S_{E^{-} D^{-}}^{n}\right)$ are both 0 , and there exists no obvious difference, thus Table 2 skips over them. Significances between other gene population and non-essential disease gene population are figured out by the rank sum test. In view of Table 2, $M d\left(S_{E^{-} D^{-}}^{n}\right)$ are overwhelmingly significantly smaller than $M d\left(S_{E^{-} O}^{n}\right)$. It illustrates that disease genes are weakly connected with essential genes within the protein interaction network.

Motivated by Goh et al.'s work [29], the above conclusion can be explained similarly. Provided disease genes are strongly associated with essential genes, mutations of disease genes are likely to affect essential genes seriously and cause critical diseases or even death. Thus, individuals whose disease genes tightly interacted with essential genes should have been eliminated during the course of evolution. In one word the existing protein-protein interaction network structure can protect the primary normal functions of life.

Table 2: Median values of the strengths of topology associations between $X$ and nondisease essential genes $(E)$ in the $n(n \in 1,2, \ldots, 5)$ neighbors.

\begin{tabular}{|l|c|c|c|}
\hline & $X=D^{-}$ & $X=O$ & $\mathrm{p}$-value \\
\hline$M d\left(S_{E^{-} X}^{1}\right)$ & 0.1726 & 0.2357 & $3.6701 \mathrm{e}-22$ \\
\hline$M d\left(S_{E^{-} X}^{2}\right)$ & 0.1997 & 0.2031 & $7.7469-13$ \\
\hline$M d\left(S_{E^{-} X}^{3}\right)$ & 0.1220 & 0.1338 & 0.0047 \\
\hline$M d\left(S_{E^{-} X}^{4}\right)$ & 0.0238 & 0.0265 & 0.0260 \\
\hline$M d\left(S_{E^{-} X}^{5}\right)$ & 0.0186 & 0.0174 & 0.0050 \\
\hline
\end{tabular}




\subsection{Disease gene prediction for 110 diseases}

Generally, the main strategies for gene prioritization include local distance measurements and global distance measurements. Local distance measurements, such as $D N$ or $S P$, are simple and efficient but behave not good enough. By taking full advantages of global topological structure, global distance measurements are superior to local ones. However, the traditional global distance measurements, such as random walk with restart or kernel diffusion, may misidentify hub proteins, which are probably essential proteins, to potential disease proteins. One main reason is that the existing methods base on the hypothesis 1. In the light of $\mathrm{Wu}$ et al. [15], the hypothesis 2 and the effective novel global distance measurement for disease gene prediction have been designed. Further, this paper attempts to integrate the hypothesis 3 so as to avoid misguiding hub proteins for potential disease proteins in weighted protein interaction network as far as possible.

As shown in Supplementary Table $\mathrm{S} 1, N P_{D \& E}$ ranks all of the disease genes of 16 diseases first (Enrichment Score is 50), such as Alzheimer Disease (4 disease genes), Fanconi anemia (10 disease genes), etc. Table 3 summarizes the performance of $N P_{D \& E}$ and four competing methods on 110 diseases. Significances between our approach and four competing methods are listed in Table 4 , which are calculated by one-tailed student's $t$-test. Obviously, global distance measurements are superior to local distance measurements. The assumption of $D N$ is the test gene directly interacting with the training set, while $r W R A$ suits the test gene belonging to 2 neighbors of the training set. Although $S P$ works well in a connected network, it neglects the indirect interactions dense between disease proteins. Different with the three local distance measurements, $N P_{D}$ and $N P_{D \& E}$ not only function in most of cases but also take full advantage of global topological structure. Therefore, the performance of global distance measurements is better than local distance measurements in whole. With taking hypothesis 3 into account, the performance of $N P_{D \& E}$ increases by $9.2 \%$ compared to that of $N P_{D}$.

According to Köhler et al.'s work [11], as shown in Table 3, we divide 110 diseases into three classes including monogenic diseases (84 diseases), complex diseases (12 diseases) and cancers (12 diseases). We can see $N P_{D \& E}$ is significantly better than four competing methods on monogenic diseases, whereas similar with them on complex diseases and cancers. One reasonable explanation is some of diseases genes associated with complex diseases and cancers are also essential. There exist strong topological associations among essential proteins, which probably affect the capacity of $N P_{D \& E}$. Addition- 
ally, as shown in Figure 2, $N P_{D \& E}(84.74 \%)$ performs best under $A U C$ index, which further verifies the effectiveness and potential of the new measurement.

Table 3: The performance (the average values of Enrichment Score) of disease gene prediction

\begin{tabular}{|c|c|c|c|c|c|}
\hline & $D N$ & $r W R A$ & $S P$ & $N P_{D}$ & $N P_{D \& E}$ \\
\hline Monogenic & 27.0003 & 24.9905 & 24.0769 & 27.3403 & 30.3147 \\
\hline Complex & 9.0003 & 13.0808 & 11.5834 & 12.2199 & 13.0571 \\
\hline Cancer & 21.5427 & 25.2266 & 25.6203 & 23.2049 & 22.2861 \\
\hline All & 24.4178 & 23.7053 & 22.8714 & 25.2204 & 27.5309 \\
\hline
\end{tabular}

Table 4: One tailed $t$-tests for Table 3: $N P_{D \& E}$ versus Competing Approaches.

\begin{tabular}{|c|c|c|c|c|}
\hline & $D N$ & $r W R A$ & $S P$ & $N P_{D}$ \\
\hline Monogenic & 0.0038 & $1.6537 \mathrm{e}-05$ & $1.5453 \mathrm{e}-09$ & $2.4777 \mathrm{e}-07$ \\
\hline Complex & $9.5084 \mathrm{e}-04$ & 0.9753 & 0.4576 & 0.5575 \\
\hline Cancer & 0.7869 & 0.2135 & 0.0911 & 0.6668 \\
\hline All & 0.0012 & $1.9679 \mathrm{e}-04$ & $2.0790 \mathrm{e}-07$ & $1.7723 \mathrm{e}-05$ \\
\hline
\end{tabular}




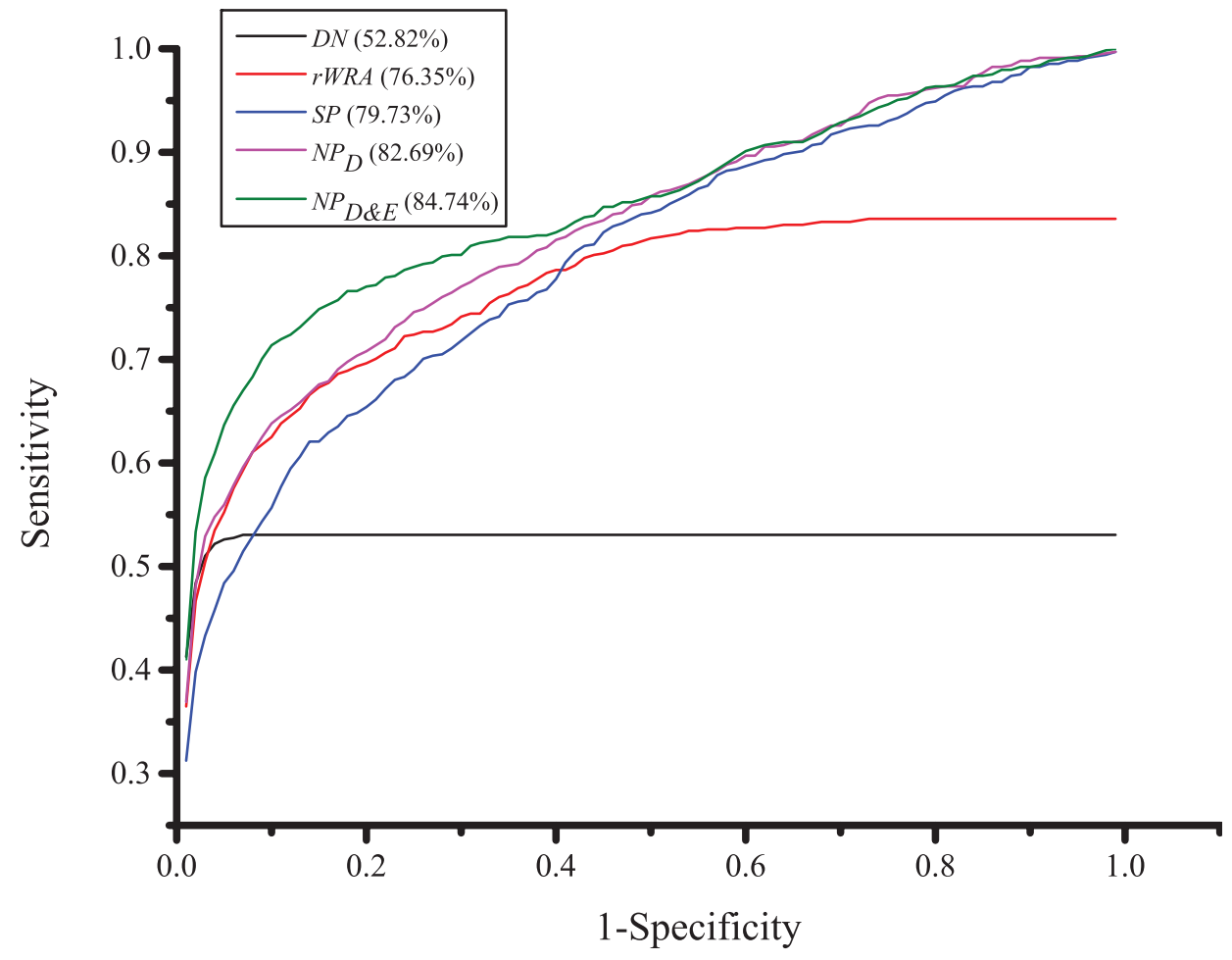

Figure 2: ROC curves.

\subsection{The role of weak links for Gene Prioritization}

This paper introduces a parameter $\beta$ to control the relative contributions of weak links to the performance of gene prioritization. The parameterdependent index for the flow pumped from node $v_{i}$ to its direct neighbor node $v_{j}$ is $N\left(v_{i}, v_{j}\right)=w_{i j}^{\beta} /\left(\sum_{k \in Q_{i}^{1}} w_{i k}^{\beta}\right)$. When $\beta$ equals zero, the index degenerates to the unweighted cases. When $\beta$ equals one, the index is equivalent to the simply weighted cases. When $\beta$ larger than one, the importance of strong links will be enhanced rather than weak links; when $\beta$ less than one, exactly the reverse. We analyzes the effects of weak links on disease gene prediction by adjusting $\beta$.

Figure 3 manifests the comparisons of the new global measurement $N P_{D \& E}$ with the traditional global measurement $N P_{D}$. No matter what value $\beta$ takes, $N P_{D \& E}$ acts markedly better than $N P_{D}$ for gene prioritization, which exactly validates the effectiveness and virtues of hypothesis 3. The performances of $N P_{D \& E}\left(\left.A U C_{N P_{D \& E}}\right|_{\beta=1}=84.74 \%\right)$ and $N P_{D}\left(\left.A U C_{N P_{D}}\right|_{\beta=1}=82.69 \%\right)$ with 
weighted protein interaction network $(\beta=1)$ are slightly better than those $\left(\left.A U C_{N P_{D \& E}}\right|_{\beta=0}=84.69 \%,\left.A U C_{N P_{D}}\right|_{\beta=0}=82.16 \%\right)$ with unweighted protein interaction network $(\beta=0)$. It emphasizes the strengths of protein interactions are beneficial to gene prioritization. Last but not least, the optimal value of $\beta$ for $N P_{D \& E}$ is $-0.59\left(\beta_{N P_{D \& E}}=-0.59\right)$, while 3.80 for $N P_{D}$ $\left(\beta_{N P_{D}}=1.51\right)$, which means $N P_{D \& E}$ productively utilizes more weak links rather than $N P_{D}$, and weak links play more valuable role than strong links in the weighted protein interaction network.

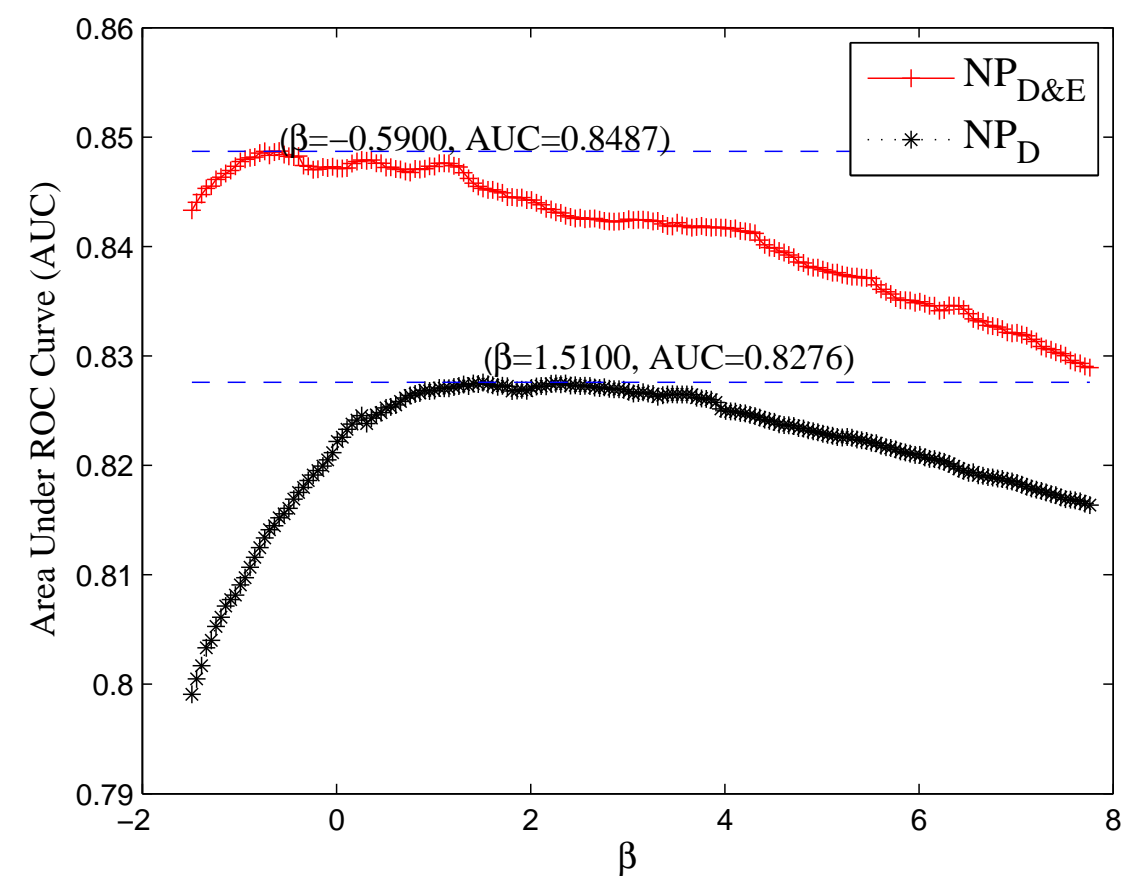

Figure 3: $A U C$ as a function of $\beta$ for disease gene prediction.

\section{Discussion and conclusion}

In recent years, utilization and integration of various Omics data have been one of the most important research for disease gene prediction, especially the protein interaction network [38-40]. Wu et al. [39] developed a method named Networked Gene Prioritizer to prioritize cancer-associated genes by integrating gene expression data and protein interaction network. 
Zhao et al. [40] proposed a katz-centrality based approach for gene prioritization through combining gene expression data and protein interactions. This paper aims to deeply understand the mechanics of protein interaction network and designs an effective novel method to discover disease genes. Recently, the applications of weighted protein interaction network have drawn increasingly attention. For instance, Itan et al. [31] presented "Human Gene Connectome" grounded on weighted protein interaction network for morbid allele discovery; inspired by the solution of the most reliable route problem in communication networks, Zhao et al. [32] designed novel local similarity indices to supplement missing protein interactions and their confidence scores. Unfortunately, topological analyses of weighted protein interaction network are still rare yet.

Exploring the properties of protein interaction network is helpful to understand disease mechanism and guarantee significance for disease gene prediction. A great deal of work has studied the characteristics of disease proteins by comparison analyses between disease and other proteins. Topological importance of disease proteins is a controversial issue. Initially, massive empirical analyses demonstrated that disease proteins were topologically important [26, 33, 34]. However, Goh et al. [29] indicated there existed a few essential proteins among disease ones, which might affect analysis results. Their research showed that non-essential disease proteins occupied topologically neutral positions too. Nevertheless, Goh et al. selected mouse lethal genes as human essential genes. With only about $10 \%$ of mouse genes knocked out, it was impossible to determine so far whether about $60 \%$ of human disease genes were essential genes [35]. After improving the above situation, several research suggested that non-essential disease proteins were topologically more important $[8,35]$. However, supposing disease proteins occupied topologically important positions, it was probably to hinder plenty of normal functions after disease genes mutated and lead to eventual deletion from the population gradually [29]. Therefore, the reasonability about topological importance of disease proteins deserves more discussion. Lately, $\mathrm{Wu}$ et al.'s empirical analysis demonstrated that disease proteins were not well connected with essential proteins [15]. Furthermore, this paper reveals that disease genes are weakly correlated with essential genes in topological association. The above two conclusions could explain the new problem to some extent. Since disease genes are weakly correlated with essential genes, mutations of disease genes will not affect the main normal activities seriously.

According to the inferences mentioned above, this paper extends network 
propagation model to integrate hypothesis 1 and hypothesis 3, and designs a novel global distance measurement for weighted protein interaction network. Experimental results verify the performance and potential of the proposed measurement, especially on monogenic diseases. In addition, we find weak links play an important role in gene prioritization, which implies weak tie effect also exists in molecular networks. Our approach will provide new insight for disease gene prediction and offer some inspirations to many research tasks, such as molecular diagnosis [31] and biomarker identification [36].

\section{Acknowledgments}

This work is partially supported by the National Natural Science of China (No. 41506198), the National Natural Science of China (No. 41476101), the National Natural Science of China (No. 11501314), the National Natural Science of China (No. 61503208), the National Science Foundation of Shandong Province (No. ZR2014AM019) and the Ministry of education of Humanities and Social Science project (No.15YJC860001).

\section{References}

[1] Y. Moreau, L. C. Tranchevent. Nature Reviews Genetics $13(8)(2012) 523-536$.

[2] M. Kussmann, S. Blum. Endocrine, Metabolic \& Immune DisordersDrug Targets (Formerly Current Drug Targets-Immune, Endocrine \& Metabolic Disorders) 7(4)(2007)271-287.

[3] J. Xu, Y. Li. Bioinformatics 22(22)(2006)2800-2805.

[4] A. Smalter, S. F. Lei, X. Chen. IEEE Interational Conference on Bioinformatics and Biomedicine (2007)209-216.

[5] P. Yang, X. L. Li, J. P. Mei, C. K. Kwoh, S. K. Ng. Bioinformatics 28(20)(2012)2640-2647.

[6] T. P. Nguyen, T. B. Ho. Artificial intelligence in medicine 54(1)(2012)6371.

[7] P. Yang, X. L. Li, H. N. Chua, C. K. Kwoh, S. K. Ng. PloS one 9(5)(2014)e97079. 
[8] S. Wu, F. Shao, R. Sun, Y. Sui, Y. Wang, J. Wang. Physica A: Statistical Mechanics and its Applications 398(2014)217-228.

[9] A. Yousef, N. M. Charkari. Journal of Theoretical Biology 383(2015)1219.

[10] S. Aerts, D. Lambrechts, S. Maity et al. Nature biotechnology $24(5)(2006) 537-544$.

[11] S. Köhler, S. Bauer, D. Horn et al. The American Journal of Human Genetics 82(4)(2008)949-958.

[12] J. Chen, B. J. Aronow, A. G. Jegga. BMC bioinformatics 10(1)(2009)73.

[13] S. Navlakha, C. Kingsford. Bioinformatics 26(8)(2010)1057-1063.

[14] J. P. Gonçalves, A. P.Francisco, Y. Moreau et al. PloS one $7(11)(2012) \mathrm{e} 49634$.

[15] S. Wu, F. Shao, J. Ji et al. PloS one 10(2)(2015)e0116505.

[16] X. Wang, N. Gulbahce, H. Yu. Briefings in functional genomics 10(5)(2011)280-293.

[17] A. Zanzoni, M. Soler-López, P. Aloy. FEBS letters 583(11)(2009)17591765.

[18] A. L. Barabási, N. Gulbahce, J. Loscalzo. Nature Reviews Genetics 12(1)(2011)56-68.

[19] M. Oti, B. Snel, M. A. Huynen et al. Journal of medical genetics 43(8)(2006)691-698.

[20] X. Wu, R. Jiang, M. Q. Zhang et al. Molecular systems biology $4(1)(2008) 189$.

[21] P. Yang, X. Li, M. Wu et al. PloS one 6(7)(2011)e21502.

[22] L. Lü, T. Zhou. Europhysics Letters 89(1)(2010)18001.

[23] P. Csermely. Trends in biochemical sciences 29(7)(2004)331-334. 
[24] Online Mendelian Inheritance in Man, OMIM(TM). McKusick-Nathans Institute of Genetic Medicine, Johns Hopkins University (Baltimore, MD) and National Center for Biotechnology Information, National Library of Medicine (Bethesda, MD), 2014. World Wide Web URL: http://www.ncbi.nlm.nih. gov/omim/.

[25] C. W. Chang, W. C. Cheng, C. R. Chen et al. PloS one 6(7)(2011)e22859.

[26] Z. Tu, L. Wang, M. Xu et al. BMC genomics 7(1)(2006)1-13.

[27] A. Franceschini, D. Szklarczyk, S. Frankild et al. Nucleic acids research 41(D1)(2013)D808-D815.

[28] P. Symeonidis, E. Tiakas, Y. Manolopoulos. Proceedings of the fourth ACM conference on Recommender systems (2010)183-190.

[29] K. I. Goh, M. E. Cusick et al. Proceedings of the National Academy of Sciences 104(21)(2007)8685-8690.

[30] R. A. George, J. Y. Liu, L. L. Feng et al. Proceedings of the National Academy of Sciences 104(21)(2007)8685-8690.

[31] Y. Itan, S. Y. Zhang, G. Vogt et al. Proceedings of the National Academy of Sciences 110(14)(2013)5558-5563.

[32] J. Zhao, L. Miao, J. Yang et al. Scientific reports 5(2015)2261.

[33] S. Wachi, K. Yoneda, R. Wu. Bioinformatics 21(23)(2005)4205-4208.

[34] P. F. Jonsson, P. A. Bates. Bioinformatics 22(18)(2006)2291-2297.

[35] J. E. Dickerson, A. Zhu, D. L. Robertson et al. PloS one $6(11)(2011) \mathrm{e} 27368$.

[36] H. Y. Chuang, E. Lee, Y. T. Liu et al. Molecular systems biology $3(1)(2007) 1$.

[37] O. Vanunu, O. Magger, E. Ruppin et al. PLoS Computational Biology 6(2010)0641.

[38] R. G. Gudivada, X. A. Qu, J. Chen et al. Journal of biomedical informatics 41(5)(2008)717-729. 
[39] C. Wu, J. Zhu, X. Zhang. BMC bioinformatics 13(1)(2012)182.

[40] J. Zhao, T. H. Yang, Y. Huang et al. PloS one 6(9)(2011)e24306. 